\title{
Tackling Reversible Conversion Reaction Mechanism for Lithium Based Battery
}

\author{
Langli Luo ${ }^{1}$, Jinsong $\mathrm{Wu}^{1,2}$, Junming $\mathrm{Xu}^{1}$ and Vinayak P. Dravid ${ }^{1,2}$ \\ 1. NUANCE Center, Northwestern University, Evanston, IL 60208, United States \\ 2. Department of Materials Science and Engineering, Northwestern University, Evanston, IL 60208, \\ United States
}

The demand for next generation of lithium based batteries with higher capacity and rate as well as increased cycle and calendar life requires new lithium storage and transfer mechanisms beyond what current lithium ion battery technique could provide. In lithium based battery, the lithium ions are stored in electrode materials either by physically intercalating (e.g. $\mathrm{LiCoC}_{2}$ ) or chemically alloying (e.g. $\mathrm{Li}_{\mathrm{x}} \mathrm{Si}$ ). In the past decade, the conversion reaction of $3 \mathrm{~d}$ metal oxide nanostructures which involves the formation and decomposition of lithium compounds in nanoscale present an array of advantages [1]. Despite a large number of synthesis and performance studies, the underlying mechanisms involving structural and phase changes during the lithiation/delithiation processes of these nanostructures has little been tackled.

The emerging in-situ transmission electron microscopy (TEM) techniques with localized electrical measurement capabilities provide a practical platform for investigating electrochemical reactions in Li-ion battery materials by building a full or half "nano-cell" inside the TEM specimen chamber [2]. Such real-time observations of dynamic composition and microstructural evolution in the electrochemical reaction have provided many novel clues to understand the lithiation/de-lithiation mechanisms at nano or atomic-scale for several novel anode materials. Herein, we report a comprehensive morphological and structural study to tackle the conversion reaction mechanisms during the lithiation/delithiation process by using graphene sheets supported $\mathrm{Co}_{3} \mathrm{O}_{4}$ nanocubes as a model system.

The graphene supported $\mathrm{Co}_{3} \mathrm{O}_{4}$ nanocubes were synthesized by simple hydrothermal method. As shown in HAADF STEM image fig. 1A and HRTEM image in fig. $1 \mathrm{C}$, these nanocubes with a size of $\sim 5 \mathrm{~nm}$ are dispersed on graphene sheets with $\mathrm{Co}_{3} \mathrm{O}_{4}$ phase confirmed by diffraction pattern in fig. 1B. The in-situ TEM experiments were performed on a specified electrical probing TEM holder with a dual-probe design as shown in Fig. 1D, i.e. one Au rod is used as the sample holder with a small amount of $\mathrm{Co}_{3} \mathrm{O}_{4} /$ Graphene dispersed on its tip; on the other side a STM tungsten (W) probe driven by Piezo-motor capable of 3-D positioning is used to mount Li metal. The W probe tip was scratched by Li metal strip and then affixed on the TEM holder inside a glove box, then transferred to TEM column with limited exposure to air $(<5 \mathrm{~s})$, when a layer of lithium oxide was grown on the surface of Li metal acting as a solid electrolyte for the nano-cell Li-ion batteries.

Time-resolved snapshots shown in fig. 2(a) to (d) and (e) to (h) present the electrochemical lithiation and delithiation process of a cluster of $\mathrm{Co}_{3} \mathrm{O}_{4}$ nanocubes on graphene sheets, respectively. As the lithiation occurs, pristine structure of $\mathrm{Co}_{3} \mathrm{O}_{4}$ nanocubes with a size of $5 \mathrm{~nm}$ decompose to $1-2 \mathrm{~nm}$ nanoparticles embedded in a matrix. The real time observation reveals the reaction starts from the contacting point between $\mathrm{Li}_{2} \mathrm{O} / \mathrm{Li}$ and $\mathrm{Co}_{3} \mathrm{O}_{4}$-Graphene and the interface between unreacted and reacted areas is clearly seen throughout the process which indicates the lithiation process is a rate-limiting 
process which is determined by either the $\mathrm{Li}^{+}$diffusion through the surface of graphene or the reaction rate of $\mathrm{Li}+$ and $\mathrm{Co}_{3} \mathrm{O}_{4}$. In the reverse process, the as-formed Co nanoparticles embedded in $\mathrm{Li}_{2} \mathrm{O}$ matrix in fig. 2(e) didn't change in the first $60 \mathrm{~s}$ of incubation time. Then, the Co nanoparticles start to decompose into smaller nanoparticles and new nuclei form on the site of the Co nanoparticles at the same time until a saturation density of nuclei is reached as seen in fig. 2(f). Within 5 mins, the newly formed phase grew into $1-3 \mathrm{~nm}$ nanoparticles while the $\mathrm{Li}_{2} \mathrm{O}$ matrix is gradually consumed which is evidenced by that the flake-like $\mathrm{Li}_{2} \mathrm{O}$ shown in fig. 2(e) changed to clear graphene sheets in fig. 2(h) after the reaction. These observation reveals that both lithiation/delithiation processes are accompanied by the formation of new phase $\left(\mathrm{Li}_{2} \mathrm{O} / \mathrm{Co}\right.$ oxides $)$ on the site of decomposed $\mathrm{Co}_{3} \mathrm{O}_{4} / \mathrm{Co}$ nanoparticles in the matrix. The structure of $\mathrm{Co}$ nanoparticles densely embedded in $\mathrm{Li}_{2} \mathrm{O}$ matrix on graphene sheets is critical for the reverse reaction to happen because the large chemical barrier for the decomposition of $\mathrm{Li}_{2} \mathrm{O}$ is lowered by small metal nanoparticles with large surface area and catalytic properties. The graphene sheets play as a supporting materials and ion/electron transport promoter without changing their own structures.

\section{Reference:}

[1] Arico, A. S., Bruce, P., Scrosati, B., Tarascon, J.-M. \& van Schalkwijk, W. Nat Mater 4 (2005), 366. [2] Huang, J. Y. Zhong, L., Wang, C. M. et al. Science 330 (2010), 1515.
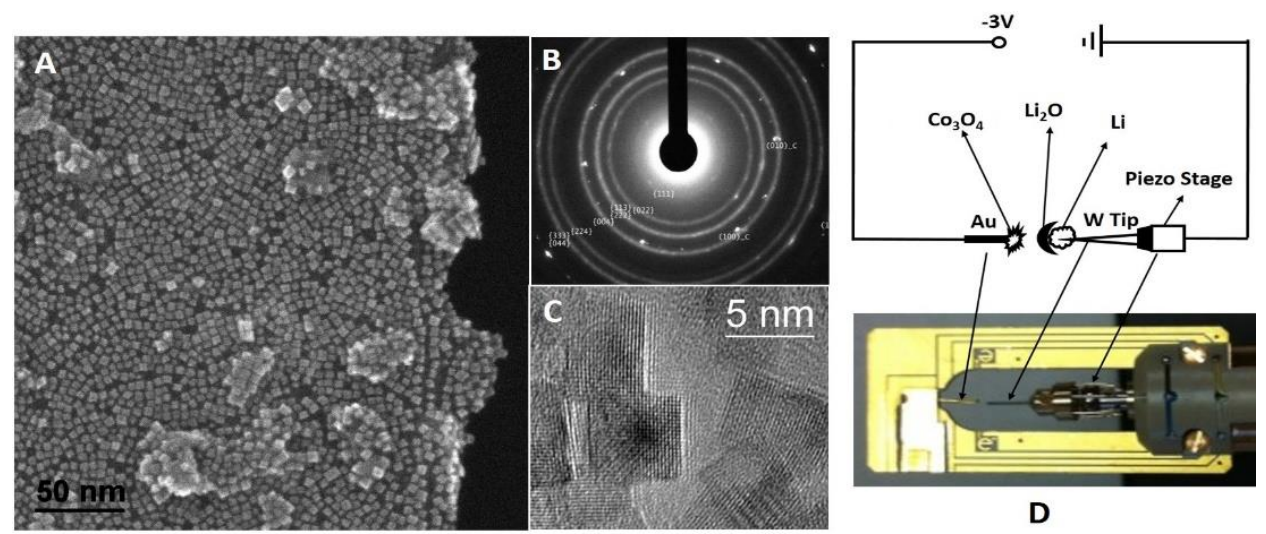

Fig. 1 As synthesized $\mathrm{Co}_{3} \mathrm{O}_{4}$ nanocubes on graphene: A. STEM Z-contrast, B. Electron diffraction, and C. HRTEM images; schematic and real layout of the TEM holder.
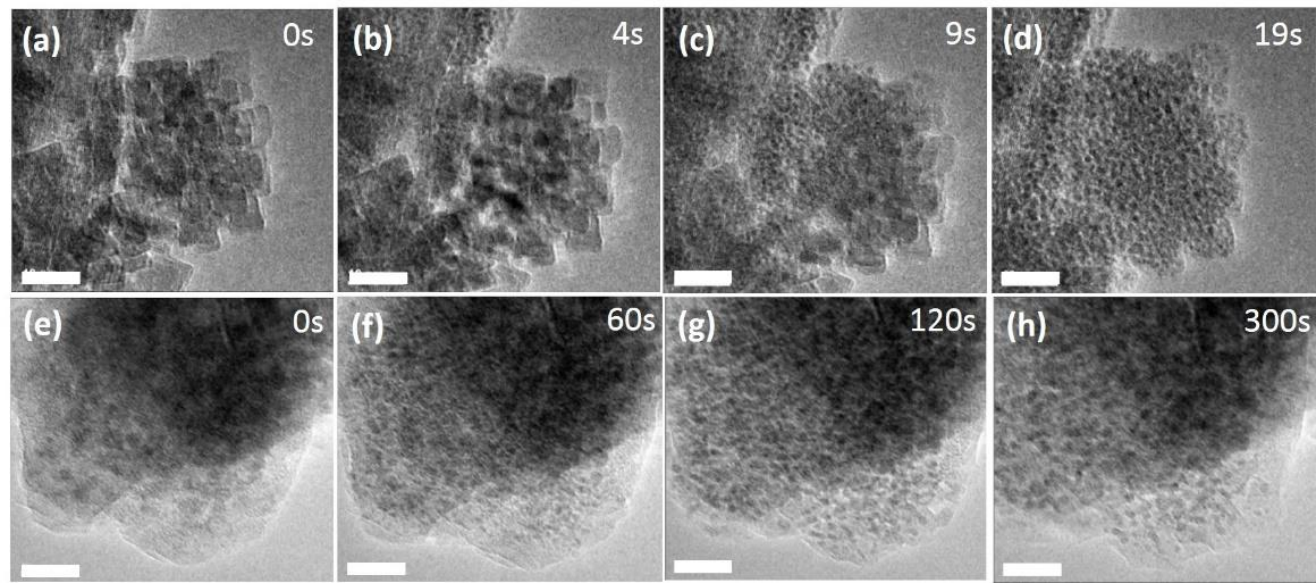

Fig.2 Time-resolved TEM images of lithiation (a-d) and delithiation (e-h) processes of $\mathrm{Co}_{3} \mathrm{O}_{4} /$ graphene. 